\title{
27 CYTOKINE SIGNATURE OF PD-1, CXCL10, AND TNF- ALPHA PREDICTS RESPONSE TO NIVOLUMAB AND IPILIMUMAB
}

${ }^{1}$ Jesper Pedersen*, 'Mateo Sokac, ${ }^{1}$ Nicolai Birkbak, ${ }^{2}$ Trine Øllegaard, ${ }^{1}$ Martin Jakobsen.

${ }^{1}$ Aarhus University, Aarhus, Denmark; ${ }^{2}$ Aarhus University Hospital, Aarhus, Denmark

Background Checkpoint inhibitors have significantly improved treatment of metastatic melanoma. Yet, $40-60 \%$ of the patients do not achieve a long-term benefit from such immunotherapy. Thus, there is an urgent need to identify biomarkers that can predict response to immunotherapy to guide patients for the best possible treatment. Here, we evaluate an unsupervised machine learning approach to identify potential cytokine signatures from liquid biopsies that predict response to immunotherapy in melanoma.

Methods Blood samples were drawn from 74 patients diagnosed with unresectable advanced-stage melanoma undergoing treatment with first-line nivolumab/ipilimumab or pembrolizumab between August 2017 - July 2019 at Aarhus University Hospital, Denmark. Blood samples were tested for plasma levels of PD-1, PD-L1, IFN-beta, IFN-gamma, CCL20, CXCL5, CXCL10, IL6, IL8, IL10, MCP1, and TNF-alpha by Meso Scale ELISA assays. Healthy controls were used to compare general cytokine levels in plasma. A bioinformatic workflow consisting of Uniform Manifold Approximation and Projection (UMAP) dimension reduction method and k-means clustering analysis was applied to define clusters based on the cytokine profile, followed by survival analysis of the clusters.

Results UMAP analysis demonstrated that the cytokine profile at baseline was similar for healthy controls and patients, regardless of treatment. Upon treatment initiation, the cytokine profile changed in a treatment-dependent way to be significantly different between patient groups. Clustering defined by the cytokine profile measured early during treatment in nivolumab/ipilimumab treated patients identified two clusters associated with superior progression-free survival (PFS) (logrank $\mathrm{p}=0.018)$. We identified that these cluster were characterized by significantly higher levels of PD-1, CXCL10, and TNF-alpha. UMAP analysis of the cytokine level as fold change over baseline level, confirmed that nivolumab/ipilimumab patients with superior PFS were characterized by higher levels of PD-1, CXCL10, and TNF-alpha. Cox regression analysis revealed high fold change of PD-1 as a strong predictor for superior PFS $(\mathrm{HR}=0.29 ; 95 \%$ CI $0.12-0.66 ; \mathrm{p}=0.0032)$. However, a similar cytokine profile was not associated to superior PFS in patients receiving pembrolizumab, suggesting that the cytokine signature is specific for nivolumab/ipilimumab treatment.

Conclusions Using unsupervised machine learning we identified a cytokine signature of high PD-1, CXCL10, and TNF-alpha to be associated with superior PFS in advanced-stage melanoma patients treated with nivolumab/ipilimumab but not pembrolizumab, with high fold change of PD-1 being a strong individual predictor for PFS.

Acknowledgements We thank the medical laboratory technicians who collected blood samples and the patients who participated in the study.

Ethics Approval The study was approved by Central Denmark Region Committees on Biomedical Research Ethics, approval number 1-10-72-374-15.

http://dx.doi.org/10.1136/jitc-2021-SITC2021.027 